\title{
Associação da doença hepática gordurosa não-alcoólica e do modelo de avaliação da homeostase de resistência à insulina
}

\author{
Association of non-alcoholic fatty liver disease and insulin resistance homeostasis \\ assessment model
}

Asociación de enfermedad hepática no alcohólica y modelo de evaluación de homeostasis de resistencia a la insulina

Josilda Ferreira Cruz ${ }^{1 *}$, Yasmim Anayr Costa Ferrari ${ }^{1}$, Tales Rabelo Alonso González ${ }^{1}$, Amanda Oliveira Barros ${ }^{1}$, Allan Victor Hora Mota ${ }^{1}$, Carla Pérez Machado², Jéssica Teles Santana ${ }^{1}$, Nathalia Nascimento Santana', Luana Rytholz Castro ${ }^{1}$, Sonia Oliveira Lima ${ }^{1}$.

\section{RESUMO}

Objetivo: Correlacionar os valores do Modelo de Avaliação da Homeostase de Resistência à Insulina (HOMAIR) com graus da esteatose hepática não alcoólica. Métodos: Estudo clínico e prospectivo, aprovado pelo Comitê de Ética através do protocolo 2.314.735 e realizado no período entre outubro de 2017 e julho de 2018, em um centro de referência em ultrassonografia em Aracaju-SE. Foram dosadas a glicemia em jejum e a insulina sérica de todos os pacientes. A seguinte fórmula foi usada para o cálculo do HOMA-IR: (glicemia em jejum $X$ insulina basal $X$ 0,0555)/22,5. Resultados: De 114 pacientes, 7 foram enquadrados nos critérios de exclusão, sendo a amostra final 107. A presença de esteatose hepática não alcoólica foi encontrada em 90 $(84,11 \%)$ pacientes. Deles, 40 correspondiam ao grau $1 ; 42$ ao grau 2 e 8 apresentaram grau 3. O HOMA-IR foi calculado e se mostrou diretamente proporcional à gravidade da esteatose hepática. $A$ ausência de infiltração gordurosa hepática teve uma média do HOMA-IR de 1,30; o grau 1 de 2,23; o grau 2 de 3,00 e o grau 3 de 8,05. Conclusão: O HOMA-IR é um parâmetro não invasivo que pode ser usado para ajudar na avaliação da gravidade da esteatose hepática não alcoólica.

Palavras-chave: Fígado gorduroso, Ultrassonografia, Resistência à insulina, Obesidade, Síndrome metabólica.

\begin{abstract}
Objective: Correlate the values of the Model of Assessment of Insulin Resistance Homeostasis (HOMA-IR) with degrees of non-alcoholic hepatic steatosis. Methods: Clinical and prospective study, approved by the Ethics Committee through protocol 2,314,735 and carried out in the period between October 2017 and July 2018, at a reference center for ultrasonography in Aracaju-SE. Fasting blood glucose and serum insulin were measured for all patients. The following formula was used to calculate HOMA-IR: (fasting blood glucose $X$ basal insulin X 0.0555) / 22.5. Results: Of 114 patients, 7 met the exclusion criteria, with the final sample being 107. The presence of non-alcoholic liver steatosis was found in $90(84.11 \%)$ patients. Of these, 40 corresponded to grade $1 ; 42$ to grade 2 and 8 had grade 3 . HOMA-IR was calculated and proved to be directly proportional to the severity of hepatic steatosis. The absence of fatty liver infiltration had an average HOMAIR of 1.30 ; 2.23 grade 1 ; grade 2 of 3.00 and grade 3 of 8.05 . Conclusion: HOMA-IR is a non-invasive parameter that can be used to help assess the severity of non-alcoholic hepatic steatosis.
\end{abstract}

Keywords: Fatty liver, Ultrasonography, Insulin resistance, Obesity, Metabolic syndrome.

1 Universidade Tiradentes (UNIT), Aracaju-SE. *E-mail: josildafcruz@gmail.com

2 Santa Casa de Misericórdia de Belo Horizonte, Belo Horizonte-MG.

SUBMETIDO EM: 5/2020

ACEITO EM: 6/2020

PUBLICADO EM: 8/2020 


\section{RESUMEN}

Objetivo: correlacionar los valores del Modelo de evaluación de la homeostasis de la resistencia a la insulina (HOMA-IR) con los grados de esteatosis hepática no alcohólica. Métodos: Estudio clínico y prospectivo, aprobado por el Comité de Ética a través del protocolo 2.314 .735 y realizado en el período comprendido entre octubre de 2017 y julio de 2018, en un centro de referencia para ecografía en Aracaju-SE. La glucosa en sangre en ayunas y la insulina en suero se midieron para todos los pacientes. La siguiente fórmula se usó para calcular HOMA-IR: (glucosa en sangre en ayunas X insulina basal X 0.0555) / 22.5. Resultados: De 114 pacientes, 7 cumplieron los criterios de exclusión, siendo la muestra final 107. La presencia de esteatosis hepática no alcohólica se encontró en 90 pacientes (84,11\%). De estos, 40 correspondían al grado 1; 42 a grado 2 y 8 tenían grado 3 . HOMA-IR se calculó y demostró ser directamente proporcional a la gravedad de la esteatosis hepática. La ausencia de infiltración de hígado graso tuvo un HOMA-IR promedio de 1.30; 2.23 grado 1; grado 2 de 3.00 y grado 3 de 8.05. Conclusión: HOMA-IR es un parámetro no invasivo que puede usarse para ayudar a evaluar la gravedad de la esteatosis hepática no alcohólica.

Palabras clave: Hígado graso, Ultrasonido, Resistencia a la insulina, Obesidad, Síndrome metabólico.

\section{INTRODUÇÃO}

A Doença Hepática Gordurosa Não Alcoólica (DHGNA) é uma doença caracterizada pelo acúmulo de gordura nos hepatócitos que excede de 5 a $10 \%$ do peso do fígado, na ausência de outras etiologias de doença hepática, como consumo de álcool, hepatites virais e doenças de depósito (CHALASANI N, et al., 2018). É apontada como a doença hepática mais prevalente em todo o mundo, causando um grande impacto na saúde pública e com prevalência entre 20 e 40\% pela ultrassonografia. Este índice é maior em grupos específicos, como em obesos mórbidos, nos quais excede 80\% (SAYINER M, et al., 2017). Em países ocidentais, foi observada uma maior prevalência, em comparação aos países orientais, considerando que 0 fator ambiental é, isoladamente, um importante fator de risco (CRUZ MAF, et al., 2015).

A gravidade da DHGNA é variável, apresentando um espectro que vai da esteatose simples, presença de gordura em mais de $5 \%$ dos hepatócitos com curso benigno, à esteato-hepatite, mais severa por estar associada à inflamação e um risco aumentado para o desenvolvimento de carcinoma hepatocelular (TARGHER G, et al., 2013). Os principais fatores de risco para a DHGNA incluem: obesidade, diabetes, idade avançada, dislipidemia, circunferência abdominal aumentada, síndrome metabólica, resistência insulínica e índice de massa corporal elevado (HEGAZY MA, et al., 2012). Além disso, a terapia prolongada (maior que seis meses) com algumas medicações como amiodarona, tamoxifeno, diltiazem, cortisona e antirretroviral induzem a DHGNA (ANDRADE GC, et al., 2016).

A DHGNA é referida como a manifestação hepática da síndrome metabólica (SM), devido a sua incidência paralela à da $\mathrm{SM}$ e a sua associação com obesidade e resistência insulínica (RI), que é o mecanismo fisiopatológico coincidente (HEGAZY MA, et al., 2012). Atualmente a RI é definida como uma condição na qual quantidades normais de insulina são inadequadas para produzir a resposta biológica esperada em tecidos-alvo, incluindo tecido adiposo, músculo e fígado. Dessa forma, os componentes da SM também aumentam o risco de desenvolver DHGNA (CHALASANI N, et al., 2018).

Diversos fatores contribuem para o desenvolvimento da esteatose hepática não alcoólica e muitos deles estão associados ao surgimento e agravamento da resistência insulínica (CSAK T, et al., 2011). Köroğlu E, et al. (2016) correlacionaram a RI com o estresse oxidativo aumentado na DHGNA e mostraram que o grau de infiltração gordurosa e o grau necroinflamatório foram significativamente maiores nos pacientes com RI, desta forma com risco aumentado de progressão da doença. Hegazy MA, et al. (2012) mostraram uma associação altamente significativa entre o nível de resistência à insulina e os graus ultrassonográficos de gravidade da esteatose hepática não alcoólica.

Vários mecanismos podem contribuir para patogênese da DHGNA, como o aumento do aporte hepático de gordura a partir da circulação plasmática, aumento da lipogênese hepática e redução da oxidação lipídica. 
As consequências desses eventos são hipertrofia e hiperproliferação dos adipócitos e resistência à insulina, que induz a secreção de substâncias pró-inflamatórias como interleucina 6 (IL-6) e fator de necrose tumoral (TNF- $\alpha$ ). Essas condições contribuem para disfunção mitocondrial e produção de espécies reativas de oxigênio (EROs), fenômenos que podem agravar a esteatose hepática para o processo de fibrose e cirrose (DEL CAMPO JA, et al., 2018).

A evolução da DHGNA ocorre como uma sequência de etapas com o aumento do estresse oxidativo e do retículo endoplasmático, da disfunção mitocondrial e endotoxemia crônica, predispondo a uma maior agressão hepatocelular (DAY CP e JAMES OF,1998). Há ainda fatores genéticos, como mutações no gene PNPLA3, que podem predispor o indivíduo a uma menor capacidade antioxidante, aumentando o risco para formas mais graves da DHGNA (FELDMAN A, et al., 2017).

$O$ tecido adiposo é o principal contribuinte de ácidos graxos livres e lipotoxicidade sistêmica para o desenvolvimento da esteatose hepática não alcoólica (KUMARENDRAN B, et al., 2018). A RI é uma condição na qual as células do corpo se tornam resistentes aos efeitos da insulina e isso é relacionado não só ao aparecimento da esteatose hepática, não alcoólica como também à sua progressão para formas mais avançadas (HEGAZY MA, et al., 2012; CRUZ MAF, et al., 2015).

O prognóstico da DHGNA depende diretamente do achado histológico no momento do diagnóstico. Sendo que, para pacientes com apenas esteatose, o risco de cirrose em 10 a 20 anos é de 1 a 2\%; já os pacientes com esteato-hepatite têm uma chance de evoluírem para cirrose de 10 a $15 \%$ em 10 anos e os pacientes que desenvolvem cirrose apresentam alto risco de morte por hepatopatias crônicas e, inclusive, carcinoma hepatocelular. O portador de DHGNA, quando comparado à população geral, tem menor sobrevida, sendo a doença cardiovascular, o câncer e a própria doença hepática as três causas que lideram a sua mortalidade (RINELLA ME, 2015).

O Homeostasis model assessment - insulin resistance ou índice do modelo de avaliação da homeostase da resistência à insulina (HOMA-IR), proposto por Matthews DR, et al. (1985), é obtido a partir do cálculo da glicemia de jejum e os nível de insulina basal. Este índice é o mais utilizado tanto na prática diária como em estudos epidemiológicos, muito embora outros parâmetros possam ser úteis para identificar RI, como o teste Quicki, o índice de sensibilidade à insulina, insulinemia de 2 horas, a diferença entre as áreas sob insulina e as curvas de glicose. O bom desempenho diagnóstico do HOMA-IR na presença de DHGNA, sugere que este é um teste de rastreio de fácil avaliação e acessível, sendo assim aplicado na prática clínica (GUTIERREZBUEY G, et al., 2017).

A biópsia hepática é considerada padrão-ouro no diagnóstico da DHGNA, tanto para diferenciar a esteatohepatite da esteatose simples como para avaliar a gravidade dos danos histológicos, entretanto, por ser um procedimento invasivo, torna-se limitado o seu uso na prática rotineira. Nesse contexto, métodos de imagem não invasivos estão sendo cada vez mais utilizados no diagnóstico e acompanhamento da DHGNA (LÉDINGHEN V, et al., 2016).

A ultrassonografia, tomografia computadorizada (TC) e ressonância magnética (RM) são os principais métodos de imagem utilizados na avaliação da esteatose hepática não alcoólica, cujo objetivo é avaliar e graduar de modo não invasivo a infiltração gordurosa no fígado (MIYAKE T, et al., 2015).

Outras modalidades de exame de imagem avaliam a elasticidade do parênquima hepático, utilizando excitação mecânica do tecido, como a Transient Elastography, também conhecida como FibroScan, o Acoustic Radiation Force Impulse (ARFI) e a Elastografia Shear Wave (YONEDA M, et al., 2010). Em estudo feito por Cassinotto C, et al. (2014) comparando a Elastografia Shear Wave, FibroScan e ARFI, constatou-se que não houve diferença no diagnóstico de fibrose avançada, porém a elastografia Shear Wave mostrou-se mais eficaz para fibroses leve e moderada.

$\mathrm{Na}$ TC o fígado normal oferece uma atenuação de 50-65 unidade hounsfield (HU), um valor de 8-10 HU a mais que o baço. No entanto, a atenuação hepática decresce para menos de $40 \mathrm{HU}$ quando há infiltração hepática de gordura (ZHOU JH, et al., 2019). Seu uso é limitado por não ter acurácia no diagnóstico das formas leves e moderadas, pela exposição do paciente a radiação ionizante e 0 alto custo do exame (LÉDINGHEN V, et al., 2016). 
A RM utiliza diversos parâmetros diagnósticos que inclui o desvio químico, saturação de gordura e espectroscopia, sendo esta última a mais sensível. Apesar da RM possuir um alto potencial para diagnóstico de DHNGA, trata-se de um exame oneroso, o que impossibilita seu uso rotineiro (YOUNOSSI ZM, 2018).

A US é disponível, menos oneroso e não expõe o paciente a radiação ionizante, sendo, portanto, o primeiro exame utilizado na investigação da DHGNA (TOVO CV, et al., 2015). Na US, a esteatose hepática não alcoólica apresenta-se como uma área hiperecóica, devido ao aumento do reflexo causado pelo acúmulo de vesículas de gordura e atenuação vascular (SHEN FF e LU LG, 2016). A graduação é feita utilizando uma escala que divide a severidade da doença em normal (grau 0), leve (grau 1), moderado (grau 2) e severo (grau 3), sempre através de uma comparação entre a ecogenicidade dos parênquimas hepático e renal (LEE $\mathrm{DH}, 2017)$. A sensibilidade e a especificidade da ultrassonografia na detecção de esteatose hepática não alcoólica pode chegar de 83 a 94\% e a 84 a 100\% respectivamente na dependência do grau de gravidade da doença (ALMEIDA MA, et al., 2008).

O HOMA-IR é relacionado aos graus de esteatose hepática não alcoólica na ultrassonografia (US) e pode ajudar na seleção de pacientes para avaliação histológica pela biópsia de fígado, que é considerada o padrãoouro para classificar a infiltração gordurosa hepática, inflamação e fibrose (HEGAZY MA, et al., 2012). A combinação de ultrassonografia do fígado e do HOMA-IR é muito importante e pode ser preditiva de severidade, já que a RI é associada a um alto grau de acúmulo de gordura no fígado em pacientes com DHGNA (CRUZ MAF, et al., 2015).

O objetivo do presente estudo é correlacionar os valores do HOMA-IR com os graus de esteatose hepática não alcoólica, com o intuito de selecionar pacientes mais vulneráveis à maior gravidade de infiltração gordurosa hepática, permitindo intervenções terapêuticas precoces, evitando piores prognósticos e maiores custos à saúde pública

\section{MÉTODOS}

\section{Desenho de Estudo}

Trata-se de um estudo clínico, prospectivo, com abordagem analítica quantitativa.

\section{Local}

Os dados foram coletados em um centro de referência de ultrassonografia no município de Aracaju - SE, Brasil, no período entre Outubro/2017 a Julho/2018. Os exames foram realizados por um único examinador, sendo este um médico com experiência no diagnóstico de esteatose hepática não alcoólica, com o mesmo aparelho de ultrassonografia e boa resolução de imagem.

\section{População do estudo}

O software $G$ * Power versão 3.1.9.2 foi usado para calcular a amostra. $O$ tamanho do efeito foi $f=0,4,0$ nível de significância $\alpha=0,05$ e o poder estatístico $1-\beta=0,90$, com uma amostra mínima de 93 pacientes, foram considerados para um teste $F$.

\section{Critérios de inclusão}

Foram incluídos pacientes de ambos os sexos, com idade entre 18 e 70 anos.

\section{Critérios de exclusão}

Pacientes com consumo de álcool $\geq 140 \mathrm{~g} / \mathrm{semana}$ (sexo masculino) e $\geq 70 \mathrm{~g} / \mathrm{semana}$ (sexo feminino); pacientes portadores de doenças hepáticas crônicas; de tumor primário no fígado; de deficiência cognitiva e em uso regular de drogas indutoras de esteatose hepática como amiodarona, tamoxifeno, corticosteroides, diltiazem e antirretroviral. 


\section{Aspectos éticos}

Foi redigido um Termo de Consentimento Livre e Esclarecido (TCLE) conforme as normas do Conselho Nacional de Saúde (CNS) explicitadas na resolução 510/16, de 7 de abril de 2016, o qual foi assinado por todos os participantes. Foi esclarecido aos mesmos que eles poderão se recusar de participar do estudo, não respondendo a alguma pergunta do questionário ou se negando à realização de exames. Os dados obtidos foram sigilosos. Este projeto foi encaminhado ao Comitê de Ética em Pesquisa (CEP) da Universidade Tiradentes, situada em Aracaju - SE, e aprovado pelo parecer 2314735.

\section{Procedimento de coleta de dados}

O procedimento da coleta foi dividido em três etapas: 1, 2 e 3:

\section{Etapa 1:}

A primeira etapa consistiu no preenchimento do TCLE e do questionário sociodemográfico, contendo as seguintes variáveis: nome, idade, sexo, consumo de álcool, ausência de doenças hepáticas crônicas ou tumores primários no fígado e uso de medicamentos.

Para se formular a hipótese diagnóstica de DHGNA o consumo de álcool semanal foi considerado $<140 \mathrm{~g} /$ semana para homens e $<70 \mathrm{~g} /$ semana para mulheres, através do questionário sociodemográfico com informações sobre hábitos etílicos. A presença e frequência de etilismo foi conhecida e o consumo foi calculado, utilizando-se a fórmula: dose em $\mathrm{ml} \times$ grau $\times 0,8 / 100$, onde os graus ou teor alcoólico das bebidas são conhecidos, a saber: cerveja 4, vinho 12, conhaque 40, rum 40, uísque 43, pinga 46 (MINCIS M e MINCIS $\mathrm{R}, 2011)$.

\section{Etapa 2:}

O exame de ultrassonografia abdominal modo $\mathrm{B}$ foi realizado posteriormente, com transdutor convexo, dinâmico (com formação da imagem contínua e automática), de frequência de $3,75 \mathrm{MHz}$. Os pacientes se encontravam com preparo adequado, ou seja, jejum de no mínimo 6 horas e uso de antiflatulento.

Nesta etapa o exame ultrassonográfico abdominal modo B, foi classificada a esteatose hepática não alcoólica em graus (SAADEH S, et al., 2002). Grau 0: Ecogenicidade normal; Grau 1: Esteatose Leve, com visualização de ecos finos do parênquima hepático, visualização normal do diafragma e dos vasos intrahepáticos; Grau 2: Esteatose moderada, com aumento difuso nos ecos finos, visualização prejudicada dos vasos intra-hepáticos e do diafragma; Grau 3: Esteatose severa, com um aumento importante dos ecos finos, visualização prejudicada ou ausente dos vasos intra-hepáticos.

\section{Etapa 3:}

Todos os pacientes foram submetidos ao exame de glicemia em jejum e insulina sérica. O Modelo de Avaliação da Homeostase de Resistência à Insulina (HOMA-IR), proposto por Matthews DR, et al. (1985), foi usado, buscando estimar a resistência insulínica (RI). A seguinte fórmula foi usada para calcular o HOMA-IR: glicemia em jejum do sangue $X$ insulina basal X 0,0555 / 22,5.

\section{Análise estatística}

Os dados foram analisados de forma descritiva e analítica, as variáveis contínuas foram observadas de acordo com a distribuição de normalidade pelo teste de Shapiro-Wilk. Como o pressuposto não foi atendido, os dados foram apresentados como mediana e os respectivos intervalos de interquartis. Foi submetido a transformação do Box-Cox e a média desse logaritmo foi usada permitindo a utilização do teste paramétrico Análise de variância (ANOVA) com correção de idade e sexo. O software usado foi o International Business Machines Corporation Statistical Package for the Social Sciences (IBM SPSS $\circledast$ ) versão 22.0 com nível de significância $p<0,05$.

\section{RESULTADOS}

Foram avaliados 114 pacientes através da ultrassonografia abdominal. Destes, 7 atingiram os critérios de exclusão, restando então, 107 para análise estatística final. A amostra final apresentou $36(34 \%)$ homens e $71(66 \%)$ mulheres, com uma mediana de idade de 50 anos. 
Em uma análise inicial, pode-se perceber que 17 (16\%) pacientes não apresentaram esteatose hepática não alcoólica; 40 (37\%) apresentaram grau 1; 42 (39\%) grau 2, e 8 (8\%) apresentaram grau 3, conforme demonstrado no Gráfico 1.

Gráfico 1 - Frequência absoluta por sexo em relação aos graus de esteatose hepática não alcoólica diagnosticada pela ultrassonografia abdominal, $n=107$. Aracaju-SE, 2020.

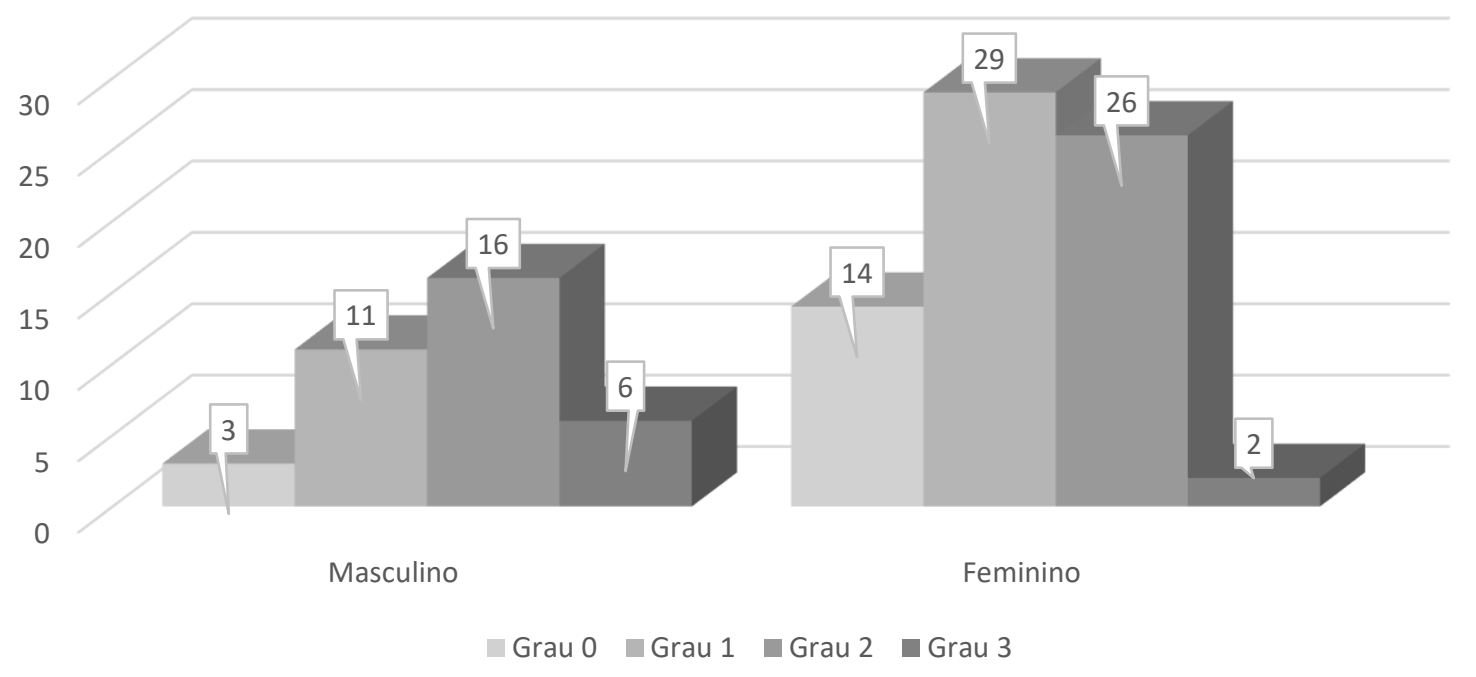

Fonte: Cruz JF, et al., 2020.

A mediana dos valores de Glicemia em jejum e Insulina Basal foram calculadas para cada grau de esteatose hepática não alcoólica. Em relação a insulina, o grau 1 de infiltração gordurosa apresentou de mediana 10,1, o grau 2, 10,6 e o grau 3, 27,2. Enquanto os pacientes sem esteatose hepática, apresentaram 6,5 de valor de mediana. Na análise da glicemia em jejum, ficou demonstrado valores de mediana de 91,5 para os pacientes com grau 1 de gordura hepática, de 96, para os pacientes com grau 2 e de 106,5 para os pacientes grau 3. Os pacientes sem esteatose obtiveram uma mediana de 91,5 para glicemia de jejum.

Na Tabela 1 foi observada a mediana do HOMA-IR e seus respectivos intervalos de interquartis para cada grau de esteatose hepática não alcoólica no sexo masculino, onde se evidenciou aumento dessa mediana com a maior progressão da infiltração gordurosa no fígado.

Tabela 1 - Percentis do HOMA-IR em pacientes do sexo masculino em relação aos graus de esteatose hepática não alcoólica diagnosticada pela ultrassonografia, n=36. Aracaju-SE, 2020.

\begin{tabular}{ccccc}
\hline & \multicolumn{3}{c}{$\begin{array}{c}\text { HOMA-IR } \\
\text { Graus Esteatose }\end{array}$} & \multicolumn{3}{c}{ Amplitude Interquartil } & p \\
\cline { 2 - 4 } & $\mathbf{2 5 \%}$ & $\mathbf{5 0 \%}$ & $\mathbf{7 5 \%}$ \\
\hline Grau 0 & 1,08 & 1,10 & 1,12 \\
Grau 1 & 1,05 & 1,45 & 2,29 & \multirow{2}{*}{0,0001} \\
Grau 2 & 2,05 & 2,70 & 4,90 & \\
Grau 3 & 3,85 & 5,60 & 8,67 & \\
\hline
\end{tabular}

Legenda: $\mathrm{p}$ : significância estatística; $\mathrm{n}$ : número de pacientes.

Fonte: Cruz JF, et al., 2020.

Na Tabela 2 a mediana do HOMA-IR com seus respectivos intervalos de interquartis também foi calculado para cada grau de esteatose hepática não alcoólica no sexo feminino. Um aumento da mediana do HOMA-IR foi nitidamente observado nos graus mais avançados de gordura no fígado. 
Tabela 2 - Percentis do HOMA-IR em pacientes do sexo feminino em relação aos graus de esteatose hepática não alcoólica diagnosticada pela ultrassonografia, n=71. Aracaju-SE, 2020.

\begin{tabular}{ccccc}
\hline \multirow{2}{*}{ Graus Esteatose } & \multicolumn{3}{c}{$\begin{array}{c}\text { HOMA-IR } \\
\text { Amplitude Interquartil }\end{array}$} & \multirow{2}{*}{$\mathbf{p}$} \\
\cline { 2 - 4 } & $\mathbf{2 5 \%}$ & $\mathbf{5 0 \%}$ & $\mathbf{7 5 \%}$ \\
\hline Grau 0 & 1,09 & 1,72 & 2,20 \\
Grau 1 & 2,09 & 2,64 & 3,42 & \multirow{2}{*}{0,0001} \\
Grau 2 & 2,05 & 2,37 & 4,30 & \\
Grau 3 & 8,46 & 9,21 & 9,96 & \\
\hline
\end{tabular}

Legenda: p: significância estatística; n: número de pacientes. Fonte: Cruz JF, et al., 2020.

A associação entre HOMA-IR e graus de esteatose hepática não alcoólica foi estatisticamente significativa $(p<0,0001)$.

\section{DISCUSSÃO}

A esteatose hepática não alcoólica tem preocupado a comunidade científica, considerando seu caráter assintomático, que favorece repercussões negativas independentemente do seu estágio, com risco de evoluir para carcinoma hepatocelular (KATSAGONI CN, et al., 2017). Nos Estados Unidos, a doença hepática silenciosa, como é chamada a DHGNA, se tornou a segunda principal indicação de transplante de fígado e a terceira causa de carcinoma hepatocelular, sendo importante um diagnóstico precoce (FAN Y, et al., 2017). Dessa forma, o HOMA-IR é um parâmetro simples e não invasivo que pode ajudar na avaliação da gravidade da esteatose hepática não alcoólica, em virtude da não especificidade das enzimas hepáticas (ZAPPAROLI M, et al., 2016).

Yki-järvinen H (2014) demostrou que a DHGNA predispõe ao Diabetes Mellitus tipo 2 (DM2)aumentando a quantidade de insulina necessária para manter a produção hepática de glicose nos níveis glicêmicos normais. Já nos pacientes com predisposição ao Diabetes, as células beta não conseguem manter a secreção de insulina por falência destas, levando à hiperglicemia. No presente estudo, constatou-se um aumento da insulina sérica e os graus da esteatose hepática não alcoólica. Parâmetro este usado na fórmula proposta por Matthews DR, et al. (1985) para o cálculo do HOMA-IR.

Pereira et al. (2015) encontraram prevalência de $42 \%$ de pacientes portadores de diabetes mellitus tipo 2 em pacientes com DHGNA. Na presente pesquisa, assim como a insulina, a glicemia se apresentou elevada nos graus mais avançados de esteatose hepática não alcoólica. Estes resultados demonstram que o aumento glicêmico pode ser considerado um fator de risco para a infiltração gordurosa do fígado.

Hegazy MA, et al. (2012) mostraram uma relação significativa entre o nível de resistência insulínica e a gravidade dos graus ultrassonográficos da esteatose hepática não alcoólica. O estudo de Köroğlu $E$, et al. (2016) mostrou que, na DHGNA, o grau de infiltração gordurosa e o grau necroinflamatório foram significativamente mais altos em pacientes com resistência insulínica. No presente estudo, uma associação estatisticamente significativa entre resistência insulínica e os graus de esteatose hepática não alcoólica foi encontrada. A resistência insulínica, dessa forma, desempenha um importante papel na fisiopatologia da DHGNA e o HOMA-IR é uma alternativa para a sua avaliação.

Parâmetros bioquímicos buscaram avaliar a gravidade da DHGNA, como enzimas hepáticas e ferritina; entretanto, são pouco específicos. Cruz MAF, et al. (2015), através de um estudo prospectivo com uma amostra de 800 pacientes, verificaram que quanto maior o grau de esteatose hepática não alcoólica, maior o HOMA-IR. Köroğlu E, et al. (2016) mostraram que a resistência insulínica na DHGNA esteve relacionada ao aumento do estresse oxidativo, analisando 21 pacientes que possuíam a doença hepática, submetidos à biópsia do fígado. O presente estudo evidenciou que o aumento do HOMA-IR foi consideravelmente proporcional à severidade da DHGNA, mostrando a sua importância quando combinado a exames de imagem não apenas para diagnosticar a esteatose hepática não alcoólica, mas também para predizer a sua progressão. 
Os processos de globalização e homogeneização de comportamentos, cada vez mais presentes na modernidade, favorecem o desenvolvimento e maior gravidade da DHGNA. Em vigência de incertezas nas opções terapêuticas, um exame não invasivo, rápido e de fácil acesso, como a ultrassonografia associada ao HOMA-IR, recurso amplamente disponível nos países desenvolvidos, oferece uma possibilidade de vigilância para essa doença hepática crônica. Permite diagnósticos precoces, alerta para a realização de intervenções multidisciplinares, haja vista que o portador de DHGNA com fibrose avançada, quando comparado à população em geral, tem menor sobrevida (CHOPRA S e LAI M, 2019).

Foi realizado um estudo observacional, transversal, porém os pacientes foram analisados em um único momento, sendo importante que estudos longitudinais possam confirmar a aplicabilidade e eficiência do HOMA-IR como preditor de gravidade da DHGNA.

\section{CONCLUSÃO}

Houve uma associação estatisticamente significativa entre os graus de esteatose hepática não alcoólica diagnosticada pela ultrassonografia e o aumento do índice HOMA-IR, o que reforça a importância do uso deste índice na prática clínica e em estudos epidemiológicos, possibilitando, inclusive, uma seleção de pacientes, com maior predisposição de gravidade, para avaliação histológica do fígado. Desta forma, a combinação da ultrassonografia e HOMA-IR torna-se um sensível e específico método não apenas para diagnóstico da esteatose hepática não alcoólica, mas também como indicador de gravidade.

\section{REFERÊNCIAS}

1. ALMEIDA MA, et al. Fatty liver disease in severe obese patients: Diagnostic value of abdominal ultrasound. World Journal of Gastroenterology, 2008; 14 (9): 1415-1418.

2. ANDRADE GC, et al. Non-alcoholic fatty liver disease (NAFLD) in different populations: A clinical and epidemiological study - sample of São José do Rio Preto. Revista da Associação Médica Brasileira, 2016; 62: 218-226.

3. CASSINOTTO C, et al. Noninvasive assessment of liver fibrosis with impulse elastography: comparisonof Supersonic Shear Imaging with ARFI and Fibroscan. Journal of Hepatology, 2014; 61 (3): 550-557

4. CHALASANI N, et al. The diagnosis and management of nonalcoholic fatty liver disease: Practice guidance from the American Association for the Study of Liver Diseases. Hepatology, 2018; 67: 328-357.

5. 5.CHOPRA S, LAI M. Management of nonalcoholic fatty liver disease in adults. UpToDate, 2018.

6. CRUZ MAF, et al. Association of the Nonalcoholic Hepatic Steatosis and Its Degrees with the Values of Liver Enzymes and Homeostasis Model Assessment-Insulin Resistance Index. Gastroenterology Research, 2015; 8: 260-264.

7. CSAK T, et al. Fatty acid and endotoxin activate inflammasomes in mouse hepatocytes that release danger signals to stimulate immune cells. Hepatology, 2011; 54 (1): 133-4.

8. DAY CP, JAMES OF. Steatohepatitis: a tale of two "hits"?. Gastroenterology, 1998; 114 (4): 842-845.

9. DEL CAMPO JA, et al. Role of inflammatory response in liver diseases: Therapeutic strategies. World Journal of Hepatology, 2018; 10 (1): 1-7.

10. FAN Y, et al. Relationship between Selected Serum Metallic Elements and Obesity in Children and Adolescent in the U.S. Nutrients, 2017; 9: 104.

11. FELDMAN A, et al. Clinical and Metabolic Characterization of Lean Caucasian Subjects with Non-alcoholic Fatty Liver. American Journal of Gastroenterology, 2017; 112 (1): 102-110.

12. GUTIERREZ-BUEZ G, et al. Is HOMA-IR a potential screening test for non-alcoholic fatty liver disease in adults with type 2 diabetes? European Journal of Internal Medicine, 2017; 41: 74-78.

13. HEGAZY MA, et al. Liver ultrasound is more sensitive in assessing the severity of nonalcoholic fatty liver disease with homeostasis model assessment-insulin resistance. Egyptian Liver Journal, 2012; 2: 41-46.

14. KATSAGONI CN, et al. Associations Between Lifestyle Characteristics and the Presence of Nonalcoholic Fatty Liver Disease: A Case-Control Study. Metabolic Syndrome and Related Disorders, 2017; 15 (2): $72-79$.

15. KÖROGOU E, et al. Role of oxidative stress and insulin resistance in disease severity of non-alcoholic fatty liver disease. The Turkish Journal of Gastroenterology, 2016; 27: 361-366.

16. KUMARENDRAN B, et al. Polycystic ovary syndrome, androgen excess, and the risk of nonalcoholic fatty liver disease in women: A longitudinal study based on a United Kingdom primary care database. PLoS Medicine, 2018; 15: 1-20.

17. LÉDINGHEN V, et al. Controlled attenuation parameter for the diagnosis of steatosis in non-alcoholic fatty liver disease. Journal of Gastroenterology and Hepatology, 2016; 31 (4):848-55.

18. LEE DH. Imaging evaluation of non-alcoholic fatty liver disease: focused on quantification. Clinical and Molecular Hepatology, 2017; 23 (4): 290-301.

19. MATTHEWS DR, et al. Homeostasis model assessment: insulin resistance and fl-cell function from fasting plasma glucose and insulin concentrations in man. Diabetologia, 1985; 28: 412-419. 
20. MIYAKE T, et al. Significance of exercise in nonalcoholic fatty liver disease in men: a community-based large crosssectional study. Journal of Gastroenterology, 2015; 7 (16): 2041-2052.

21. PEREIRA TJ, et al. Maternal obesity characterized by gestacional diabetes increases the susceptibility of rat offspring to hepatic steatosis via a disrupted liver metabolome. The Journal of Physiology, 2015; 593 (19): 3181-3197.

22. RINELLA ME. Nonalcoholic Fatty Liver Disease. Journal of the American Medical Association, $2015 ; 313$ (22): $2263-$ 2273.

23. SAADEH S, et al. The utility of radiological imaging in nonalcoholic fatty liver disease. Gastroenterology, 2002; 123 : 745-50.

24. SAYINER M, et al. Variables Associated with Inpatient and Outpatient Resource Utilization Among Medicare Beneficiaries with Nonalcoholic Fatty Liver Disease with or Without Cirrhosis. Journal of Clinical Gastroenterology, 2017; 51: 254-260.

25. SHEN FF, LU LG. Advances in noninvasive methods for diagnosing nonalcoholic fatty liver disease. Journal of Digestive Diseases, 2016; 17: 565-571.

26. TARGHER G, et al. Nonalcoholic Fatty Liver Disease and Reduced Serum Vitamin D3 Levels. Metabolic Syndrome and Related Disorders ,2013; 11 (4): 217-228.

27. TOVO CV, et al. Noinvasive imaging assessment of non-alcoholic fatty liver disease: focus os liver scintigraphy. World Journal of Gastroenterology, 2015; 21 (15): 4432-4439.

28. YONEDA M, et al. Serum Ferritin Is a Clinical Biomarker in Japanese Patients with Nonalcoholic Steatohepatitis (NASH) Independent of HFE Gene Mutation. Digestive Diseases and Sciences, 2010; 55 (3): 808-814.

29. YOUNOSSI ZM. The Epidemiology of Nonalcoholic Steatohepatitis. Clinical Liver Disease, 2018; 11 (4): $92-94$.

30. YKI-JÄRVINEN, H. Non-alcoholic fatty liver disease as a cause and a consequence of metabolic syndrome. Lancet Diabetes Endocrinology, 2014; 2: 901-910.

31. ZAPPAROLI M. Avaliação por imagem na síndrome metabólica: além da esteatose. Radiologia Brasileira, 2016; 49: 5-6.

32. ZHOU JH, et al. Noninvasive evaluation of nonalcoholic fatty liver disease: Current evidence and practice. World Journal Gastroenterology, 2019; 25 (11): 1307-1326. 